\title{
Functionalization of Carbon Nanotubes and Polymer Compatibility Studies
}

\author{
Antonio Sánchez ${ }^{1}$, Rodrigo Cué Sampedro ${ }^{1}$, Laura Peña-Parás ${ }^{2} \&$ Erika Palacios-Aguilar ${ }^{1}$ \\ ${ }^{1}$ Tecnológico de Monterrey, Eugenio Garza Sada 2501, Col. Tecnológico, Monterrey, N. L., México \\ ${ }^{2}$ Universidad de Monterrey, Ave. Morones Prieto 4500 Pte., Col. Jesús M. Garza, San Pedro Garza García, N. L., \\ México \\ Correspondence: Antonio Sánchez, Tecnologico de Monterrey, Eugenio Garza Sada 2501, Col. Tecnológico, \\ Monterrey, N. L. 64849, México. Tel: 52-818-358-2000. E-mail: asanfer@itesm.mx
}

Received: September 5, 2013 Accepted: September 18, $2013 \quad$ Online Published: October 23, 2013

doi:10.5539/jmsr.v3n1p1 URL: http://dx.doi.org/10.5539/jmsr.v3n1p1

\begin{abstract}
Multiwalled carbon nanotubes (MWNTs) were oxidated and functionalized in order to determine the structural and chemical changes on their atomic bonding. MWNTs were oxidized in an aqueous solution of $\mathrm{HNO}_{3}(70 \%)$ and $\mathrm{H}_{2} \mathrm{SO}_{4}(95 \%)$ at $25{ }^{\circ} \mathrm{C}$. Pristine and oxidized carbon nanotubes (ox-MWNTs) were characterized by scanning electron microscopy (SEM), Fourier transform infrared spectroscopy (FTIR), Raman spectroscopy, and thermogravimetrical analysis (TGA). MWNTs and ox-MWNTs were silane-functionalized and incorporated into a polymethyl-methacrylate (PMMA) matrix. The dispersion properties and interface interactions were studied for the composite materials. MWCNTs oxidation was performed and supported by Raman spectroscopy and FTIR results of disorder and defects in the carbon lattice; as well as change in the amount and type of chemical groups attached to the CNTs walls. Roughness areas visible in SEM images indicate Van der Waals interactions between CNTs and functionalization reagents. Control of the oxidation state can effectively direct molecular functionalization of a CNT sidewall. Composites showed good dispersion of filler; with the best one for oxidized-silane functionalized CNTs.
\end{abstract}

Keywords: nanocomposites, polymer/CNT composites, functionalization, oxidized, silane-functionalized

\section{Introduction}

The world of materials in nanometric scale provides exciting challenges and opportunities for chemists, physicists, biologists, and material scientists. Many conventional materials, e.g., metals, ceramics, and plastics, cannot fulfill all the requirements that new technologies seeking to solve the world's most immediate problems related to energy and environment need. There are, however, many cases in which a combination of two or more materials are able improve the performance and properties of those of their individual constituents.

Carbon nanotubes (CNTs) are one-dimensional nanostructures with extraordinary properties, such as electronic, mechanical, and chemical. Single-walled carbon nanotubes (SWNTs) consist in a graphene layer rolled into a tube. Close allotropes of SWNTs are multi-walled carbon nanotubes (MWNTs), which consist in nested SWNTs (Leonard, 2008). The orientation of a graphite plane in relation to the axis of the tube, as well as the diameter of the tube will determine many of its physical properties; nanotubes can be insulating, semiconducting, or metallic depending on these characteristics. A metallic carbon nanotube is an ideal one-dimensional conductor. Due to their unique transport properties, CNTs have a lot of potential applications in nanoelectronics. So far, many device prototypes based on carbon nanotubes have been developed, such as field-effect transistors (Tans, Verschueren, \& Dekker, 1998), single-electron transistors (Bachtold, Hadley, Nakanishi, \& Dekker, 2001), rectifiers (Yao, Postma, Balents, \& Dekker, 1999), and nonvolatile random access memory devices (Rueckes et al., 2000).

A good interfacial adhesion between the filler and the matrix is essential for an efficient load transfer in composite applications. An obstacle in the development of polymer-CNT composites lies in the difficulty of achieving a good dispersion and control the CNT orientation. Research has shown that an increase in the surface activity of fillers by the introduction of certain functional groups can significantly enhance their dispersion in the polymer (Wang, Shirley, Meikle, Whitby, \& Mikhalovsky, 2009). 
An approach of chemical modification that has been widely used in the last decades for purification and functionalization is the oxidation of CNTs. Oxidation results in the formation of carboxylic, alcohol, and ketone functional surface groups (Stobinski et al., 2010), These functional groups give the opportunity to further synthesize in the CNTs surface and attach different molecules or grafted polymers. The amount and type of surface groups attached to the CNTs strongly relies in the oxidation method used (Li et al., 2003).

The defect quantity and roughness on the surface of oxidized MWNT depends significantly on the type of acid treatment, increasing in the row: $\mathrm{HNO}_{3}<\mathrm{H}_{2} \mathrm{O}_{2}+\mathrm{H}_{2} \mathrm{SO}_{4}<\mathrm{HNO}_{3}+\mathrm{H}_{2} \mathrm{SO}_{4}$. The right choice of oxidative media is important for successful performance of functionalized MWNT in potential applications. Alteration to the nanotubes structure due to oxidative treatment significantly influences the resulting composite properties (Mazov et al., 2012).

Funtionalization of CNTs is the strategy used nowadays to improve dispersion in organic media. There exist different methods used for the functionalization of CNTs through covalent and noncovalent chemical reactions with diverse organic molecules including polymers (Chen et al., 1998; Hill, Lin, Rao, Allard, \& Sun, 2002; Holzinger et al., 2001; Sun, Fu, Lin, \& Huang, 2002). It is known that CNTs are electron-rich molecules, which allows for any electron deficient molecules to interact with them and form donor-acceptor complexes, the same way as the electron-rich molecules form $\pi-\pi$ complexes. The $\pi-\pi$ interactions of CNTs with electron-rich molecules have been used for noncovalent functionalization (Kondratyuk \& Yates Jr, 2004; Zhao, Lu, Han, \& Yang, 2003). Recently, Eklund, Murray, and co-workers and others have shown that the noncovalent interactions between CNTs with small molecules other than $\pi-\pi$ interactions lead to adsorption (Sumanasekera, Pradhan, Romero, Adu, \& Eklund, 2002).

Adsorption could also occur from molecular interactions between carbon-hydrogen groups (CH groups) and $\pi$ systems. The existence of $\mathrm{CH}-\pi$ interactions has been known for several years. Klopman et al. (Klopman, 1974) classified the $\mathrm{CH}-\pi$ interaction as a soft acid/soft base interaction using orbital energy data of acids and bases. Although the strength of $\mathrm{CH}-\pi$ interactions is only one-tenth of the hydrogen bond, their cooperative multiple interactions significantly influence many chemical and biochemical phenomena.

\subsection{Multi-Walled Carbon Nanotubes and Their Compatibility with PMMA Resin}

The fabrication of a uniform CNT reinforced polymer composite is quite challenging due to the small size and clustering nature of carbon nanotubes. Solution evaporation methods and microemulsion polymerization techniques are the most commonly used for preparing CNTs based polymer-matrix composites (Ajayan, 1999; Barraza, Pompeo, O'Rea, \& Resasco, 2002). Du et al. provided a coagulation method to produce SWNT/PMMA composites (Du, Fischer, \& Winey, 2003). This method achieves a good dispersion of SWNT in a thermoplastic polymer matrix.

Typical mixing techniques include melt mixing and solution mixing. However, since the surface characteristics of the CNTs differ markedly from those of the PMMA, the material may exhibit phase separation after several time. In this work, siloxane chains are added to the sidewall of MWNTs, to promote their dispersion in the solvent as well as in the PMMA resin.

\section{Method}

\subsection{Materials}

All carbon nanotubes used in this study were MWNTs (CM-95), purchased from Aldrich, prepared by a chemical vapor deposition method. The range in diameter was $6-13 \mathrm{~nm}$; the length of the tubes was $5 \mu \mathrm{m}$; and the purity was greater than 95\%. Triethoxy(octyl)silane with a purity of 97.5\% (Aldrich) and 3-glycidoxypropyltrimethoxysilane with a purity of $98 \%$ (Aldrich) were used as the silane functionalization agents. The reagents used for the acid treatment were nitric acid (70\%, Aldrich), sulfuric acid (95-98\%, Aldrich), acetone $(99.5 \%$, Aldrich), and ethanol (99.5\%, Aldrich). The polymer matrix used was PMMA (Plastiglas de México S.A. de C.V.), with a modulus of elasticity of $3.2 \mathrm{GPa}$.

\subsection{Functionalization of CNTs}

Surface modification of the MWCNTs was performed through two steps: oxidation and silanization. Five different samples were prepared, including functionalization with and without acid treatment for comparison.

Unmodified CNTs $(2.5 \mathrm{~g})$ were dispersed in $120 \mathrm{ml}$ of acid solution $\left(90 \mathrm{ml}\right.$ of $\mathrm{H}_{2} \mathrm{SO}_{4}$ and $30 \mathrm{ml}$ of $\left.\mathrm{HNO}_{3}\right)$ at room temperature with $24 \mathrm{~h}$ of continuos agitation. After the oxidation process, the mixture was thoroughly washed with deionized water to remove the unreacted acid until a $\mathrm{pH}$ value of 7 was reached. The resulting oxidized CNTs (CM-95-NS) were dried in vacuum at $80{ }^{\circ} \mathrm{C}$ for $12 \mathrm{~h}$. Silanization consisted in the dispersion of 
$2.0 \mathrm{~g}$ of oxidized CNTs in a $2 \%$ silane solution diluted in $100 \mathrm{ml}$ of an ethanol-water (95:5) solution. The mixture was continoulsy stirred at $50{ }^{\circ} \mathrm{C}$ for $20 \mathrm{~h}$. Silanized CNTs were separated by filtration using distilled water and acetone, then dried at $80^{\circ} \mathrm{C}$. Silanization of CNTs without oxidation treatment was conducted in the same way as for oxidized CNTs. The code name of each CM-95 functionalization obtained can be seen in Table 1.

Table 1. Code name of samples obtained in this study

\begin{tabular}{llll}
\hline CNTs & Oxidation & Reactive of functionalization & Code name \\
\hline CM-95 & - & - & CM-95 \\
CM-95 & - & 3-Glycidoxypropyltrimetoxysilane & CM-95-G \\
CM-95 & - & trietoxy(octyl)silane & CM-95-O \\
CM-95 & Acid solution & - & CM-95-NS \\
CM-95 & Acid solution & 3-Glycidoxypropyltrimetoxysilane & CM-95-NSG \\
CM-95 & Acid solution & trietoxy(octyl)silane & CM-95-NSO \\
\hline
\end{tabular}

\subsection{Characterization}

\subsubsection{Raman Spectroscopy}

Raman spectroscopy is one of the most powerful tools for the study of carbonaceous materials, especially sp ${ }^{2}$ or close to $\mathrm{sp}^{2}$ carbon materials such as graphite, carbon nanotubes, and graphene (Meyer, Etchegoin, \& Le Ru, 2010). Raman measurements were conducted with a Senterra Raman Microscope system in a backscattering geometry. In order to preferentially probe semiconducting or metallic CNTs and thus be able to distinguish any different behavior due to electronic structure, one laser excitation, wavelength $532 \mathrm{~nm}$ excitation (corresponding to $1.0 \mathrm{~mW}$ ), was used.

\subsubsection{FTIR Spectroscopy}

Both the untreated CNT and treated CNT were characterized using a Nicolet 550 FTIR spectrometer in the range of $4000-400 \mathrm{~cm}^{-1}$. All FTIR samples were prepared with potassium bromide $(\mathrm{KBr})$.

\subsubsection{Thermal Methods}

Thermal techniques were used to evaluate the amount of heat absorbed by the sample in relation to its temperature. In this study, three different methods are used: Differential Scanning Calorimetry (DSC), Differential Thermal Analysis (DTA) and Thermogravimetric analysis (TGA). DTA (Differential Thermal Analysis) measurements were carried out on a Netzsh STA Thermobalance 409. The TG/DTA curves were

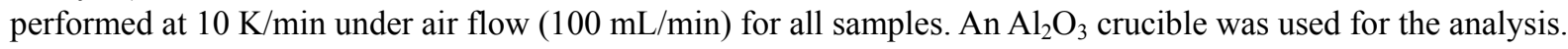

TGA for each sample was done using a TA Instruments Q500 thermal analyzer. The instrumental conditions were as follows: heating rate of $10{ }^{\circ} \mathrm{C} / \mathrm{min}$; sample mass of $8 \mathrm{mg}$ (approx.); $\mathrm{N}_{2} \mathrm{AP}(50 \mathrm{ml} / \mathrm{min}$ ) atmosphere up to $600{ }^{\circ} \mathrm{C}$ and $\mathrm{O}_{2}(50 \mathrm{ml} / \mathrm{min})$ until $900{ }^{\circ} \mathrm{C}$.

\subsubsection{Scanning Electron Microscopy}

Field Emission Scanning Electron microscopy (FESEM/EDX) was used for morphological analysis on all samples. Field emission scanning electron microscope SEM FE-7401-F was set for this labor. The operating conditions were $4 \mathrm{kV}$ along with a working distance of $3 \mathrm{~mm}$.

\subsection{Preparation of MWNTs/PMMA Composites}

Surface-modified MWNTs were dispersed in Tetrahydrofuran (THF) at room temperature for around 30 min. The PMMA resin was added to the above solution and dissolved with mechanical stirring at room temperature for $60 \mathrm{~min}$. The gel-like mixtures thus obtained were poured into a mold and heated to $40{ }^{\circ} \mathrm{C}$ for $2 \mathrm{~h}$ and then cooled down to room temperature. Samples containing 5.0 wt\% MWCNTs in PMMA were prepared using $4.75 \mathrm{~g}$ of PMMA dissolved in $15 \mathrm{ml}$ of THF with $0.25 \mathrm{~g}$ of MWCNTs.

\subsection{Carbon Nanotube Alignment}

A lot of scientific effort has been held to achieve optimal and yet efficient methods to align CNTs in polymer 
matrix. This great interest is due to the remarkable anisotropic properties that aligned CNTs cause on polymer matrices. Several methods for this purpose have been described in the literature, including: mechanical stretching, electro-spinning, wet spinning, weak magnetic fields (WMF) (Ma, Wang, \& Yu, 2010), electrohydrodynamic (EHD) patterning (Goldberg-Oppenheimer, Eder, \& Steiner, 2011), strong magnetic fields (SMF, Kimura et al., 2002), electrophoresis or electric field (Murugesh, Uthayanan, \& Lekakou, 2010), and water-assisted chemical vapor (WACV) deposition (Zeng, Ci, Carey, Vajtai, \& Ajayan, 2010).

To align MWCNTs, a hollow copper coil was rolled using $80 \mathrm{~m}$ of 18 AWG and $80 \mathrm{~m}$ of 22 AWG parallel-connected enameled copper wires. The coil was then wrapped on adhesive tape, leaving a gap of $4.5 \mathrm{~cm}$ tall and $7.5 \mathrm{~cm}$ wide. $15 \mathrm{ml}$ of samples were poured into 2 ceramic dishes and slided into the coil gap using a PMMA platform to avoid direct contact. A total of 1.6 amperes were used through the coils, generating a constant magnetic field that was expected to align the MWCNTs in the polymer matrix. The samples were afterwards left drying out overnight.

Another $15 \mathrm{~mL}$ of the gel-like materials were poured into a paper cup with flat bottom so the sample would be thinner and spread apart. Samples were then placed into the coil gap. A total of 1.8 amperes were passed through the coils and left overnight. Small squares of about $4 \times 4 \mathrm{~mm}$ were cut after drying for further analysis.

\section{Results and Discussion}

It is well established that CNTs exist in many possible morphological variations, depending on the methods used for their obtention (Wildgoose, Banks, Leventis, \& Compton, 2006). The knowledge and control of their final carbon texture and crystallite size distribution are of fundamental importance while studying their covalent or non-covalent sidewall functionalization. While it might be possible to theoretically justify that CNTs with very small diameters (1-5 nm), such as SWCNTs, exhibit different chemical reactivity from that of graphite, there is no fundamental reason why MWCNTs with diameters of $>10 \mathrm{~nm}$ should behave differently, in chemical terms, from graphite (Pumera, 2009). With this purpose, "structural disorders" in CNTs related to different types of reticular imperfections at the graphitic lattice, e.g., Stone Wales defects, vacancies and divacancies (Frenkel type pairs), edge-like defects, tube kinks, interstitial defects between external and internal tubes (Amorim, Fazzio, Antonelli, Novaes, \& da Silva, 2007; Curran, Talla, Zhang, \& Carroll, 2005), are usually invoked to account for the large part of chemical and electrochemical activity on these macromolecular systems.

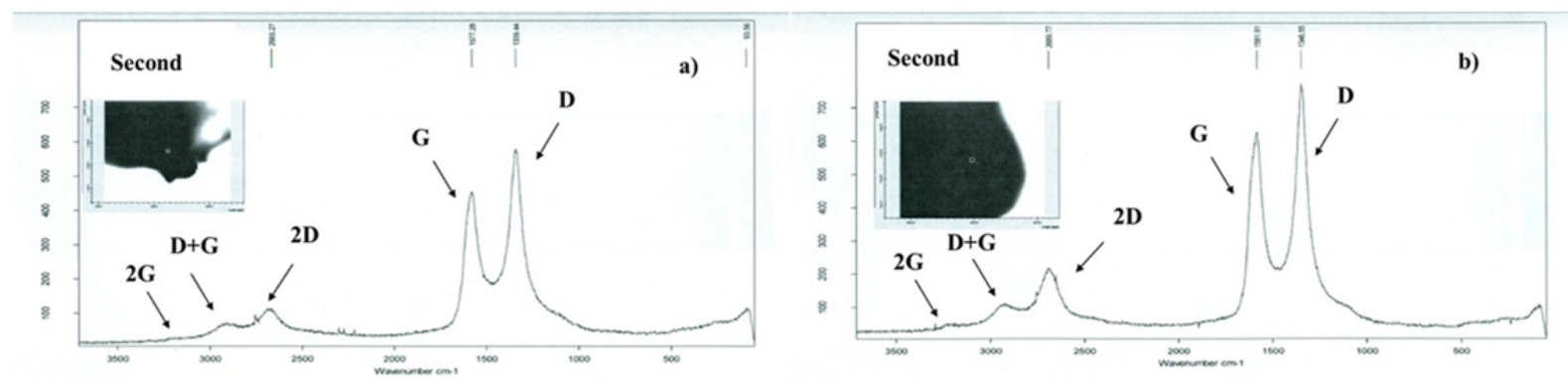

Figure 1. Raman spectra (a) CM-95; (b) CM-95-NS

The Raman spectra of pristine and oxidized MWCNTs excited with a $532 \mathrm{~nm}$ laser are shown in Figure 1. Spectra consist of the first-order $\mathrm{G}$ mode at about $1580 \mathrm{~cm}^{-1}$, which is related to $\mathrm{E}_{2 \mathrm{~g}}$ symmetry graphite mode and indicates $\mathrm{sp}^{2} \mathrm{C}-\mathrm{C}$ stretching vibrations. Also, a disorder-induced $\mathrm{D}$ band, $\mathrm{A}_{\mathrm{g}}$ symmetry, at about $1340 \mathrm{~cm}^{-1}$ is shown. The $\mathrm{D}$ band reflects the high density of states for zone-edge, which are due to the so-called double resonant Raman scattering from a nonzero-center phonon mode, and are originated from disorder and defects in the carbon lattice. The intensity ratios $\mathrm{I}_{\mathrm{D}} / \mathrm{I}_{\mathrm{G}}$ differences between the pristine MWCNTs and oxidized and functionalized samples could be attributed to an increased number of $\mathrm{sp}^{3}$ hybridized carbons (Table 2). A shift of the $\mathrm{G}$ mode is observed suggesting either a change in the amount and type of chemical groups attached to the oxidized MWCNTs walls/edges (Chiu, Duesberg, Dettlaff-Weglikowska, \& Roth, 2002), or/and increased oxygen content in the nanotubes framework that acts as p-type dopant. 
Table 2. Displacement and intensity ratios of CNTs in Raman spectroscopy

\begin{tabular}{llll}
\hline \multicolumn{1}{c}{ Sample } & D mode & G mode & $\mathrm{I}_{\mathrm{D}} / \mathrm{I}_{\mathrm{G}}$ \\
\hline CM-95 & 1339.44 & 1577.28 & 0.76 \\
CM-95-NS & 1346.55 & 1581.91 & 0.83 \\
CM-95-G & 1339.72 & 1581.39 & 0.78 \\
CM-95-NSG & 1342.29 & 1574.28 & 0.91 \\
CM-95-O & 1341.19 & 1585.01 & 0.84 \\
\hline
\end{tabular}

An FTIR spectrum for neat MWCNTs is shown in Figure 2. The characteristic vibrational modes of $\mathrm{CNT}, \mathrm{C}=\mathrm{C}$ $\left(1650 \mathrm{~cm}^{-1}\right)$ and $\mathrm{O}-\mathrm{H}\left(3400 \mathrm{~cm}^{-1}\right)$, are apparent in the spectrum. The $\mathrm{C}=\mathrm{C}$ vibrations occur due to the internal defects, and the $\mathrm{O}-\mathrm{H}$ vibration is associated with the amorphous carbon because amorphous carbon easily forms a bond with atmospheric air. Then, the intensity of this $\mathrm{O}-\mathrm{H}$ peak is relatively large and shows that a higher amount of amorphous carbon is formed during growth.

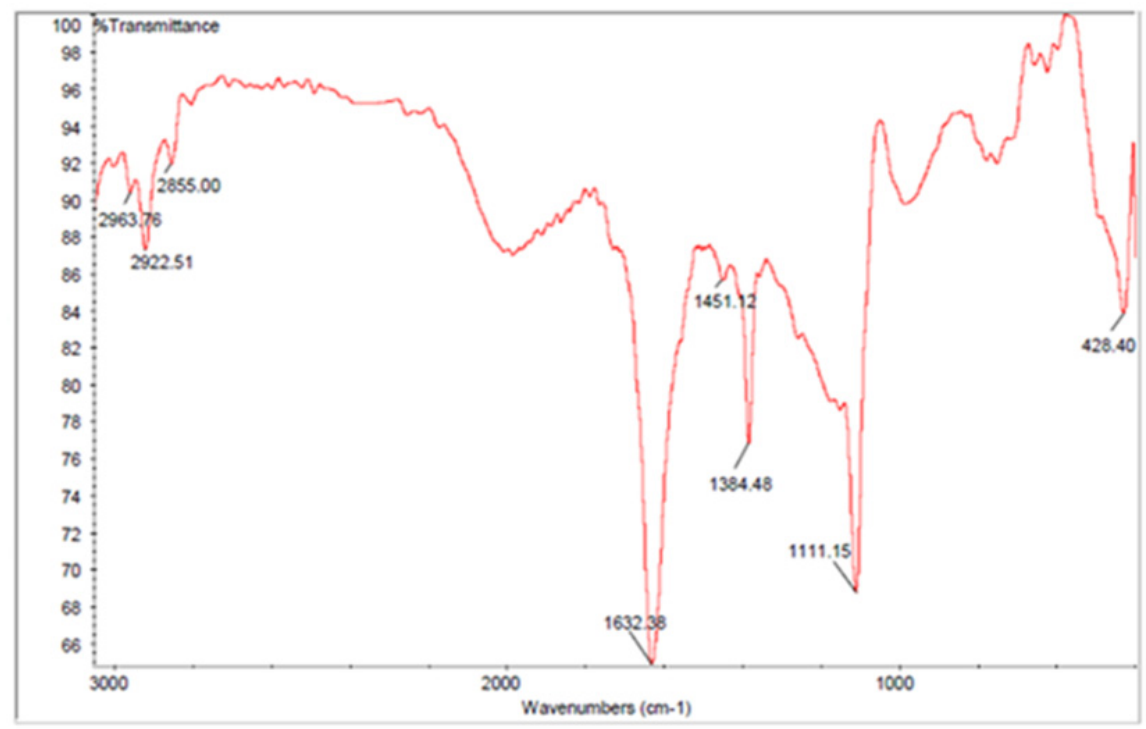

Figure 2. Infrared spectrum of neat MWCNTs

As has been experimentally (Gomez-Navarro et al., 2005; Terrones, Terrones, Banhart, Charlier, \& Ajayan, 2000) and theoretically (Amorim et al., 2007; Zhou, Wu, Duan, \& Gu, 2007) shown, local defects can modify the transport properties of CNTs. The double vacancy (DV), produced by removing two neighboring $\mathrm{C}$ atoms in a CNT, is one of the most important defects. In the experiment of Gomez-Navarro et al. (Gomez-Navarro et al., $2005)$ it was found that a low density $(0.03 \%)$ of DV could cause an increase in the resistance of a SWCNT of $400 \mathrm{~nm}$ length as large as three orders of magnitude.

Another important effect on the electron transport of CNTs comes from the adsorption of oxygen molecules $\left(\mathrm{O}_{2}\right)$, since $\mathrm{O}_{2}$ are present in the environment and oxygen is an important dopant in CNTs, which can tune their transport properties. So far, there have been extensive experimental (Collins, Bradley, Ishigami, \& Zettl, 2000; Shim, Back, Ozel, \& Kwon, 2005) and theoretical (Chan, Chen, Gong, \& Liu, 2003; Giannozzi, Car, \& Scoles, 2003; Jhi, Louie, \& Cohen, 2005) studies carried out to understand the effect of oxygen adsorption.

An oxygen molecule is adsorbed in the middle on a $(5,5)$ SWCNT with a double vacancy (CNTDV). There are two typical structures for $\mathrm{O}_{2}$ adsorption on CNT. One corresponds to physisorption at the double vacancy (type-I structure) and the other corresponds to chemical bonding with the carbon atoms (i.e., chemisorption, type-II structure), which is equivalent to replacing two carbon atoms in the perfect CNT with the two oxygen atoms (Jhi et al., 2005). The physisorption has only minor effects on the transport while the chemisorption can improve it and the resulting conductance is affected by the orientation of the $\mathrm{O}_{2}$ bonding. Figure 3 and Figure 4 represent all types of functional groups that can be obtained by oxidation under the $\mathrm{HNO}_{3} / \mathrm{H}_{2} \mathrm{SO}_{4}$ mixture. 


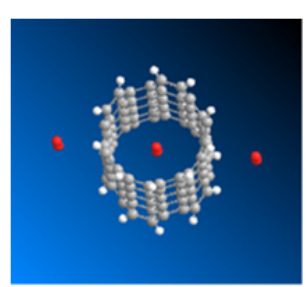

Physisorption
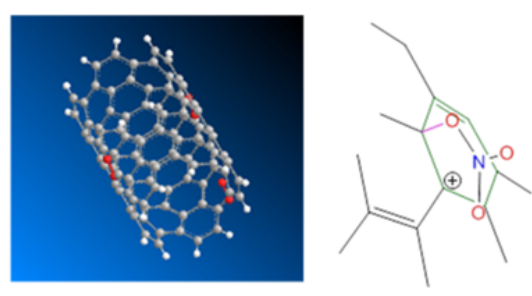

Chemisorption

Figure 3. $\mathrm{CNT}$ and $\mathrm{NO}_{3}{ }^{-}$ion interaction

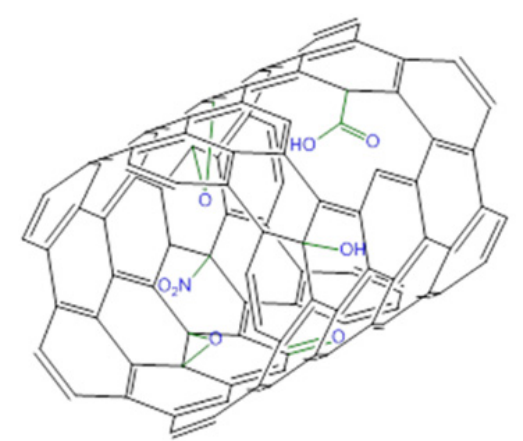

Figure 4. Broken surface of the CNT as a result of the oxidative reaction

At an atomistic level, two different mechanisms might contribute to the initiation of MWCNTs oxidation. The applied electrochemical potential depletes electro charge away from the CNTs into the bulk electrolyte. Simultaneously, individual $\mathrm{NO}_{3}{ }^{-}$molecules are attracted to the CNTs sidewall and oxidize it via accumulating local charge transfer.

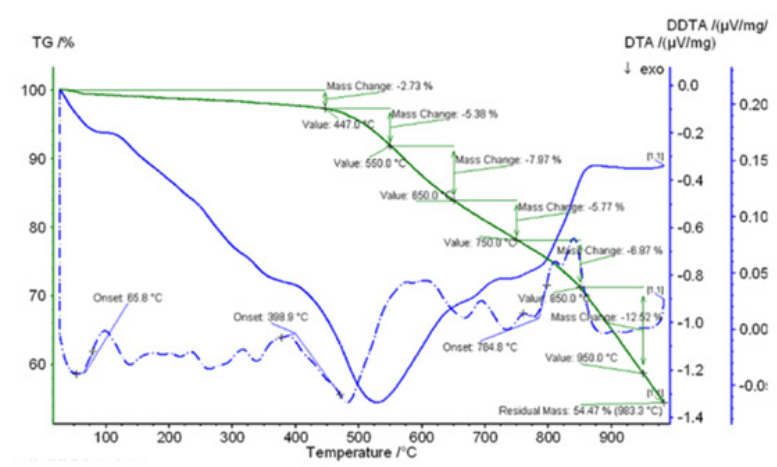

CM-95

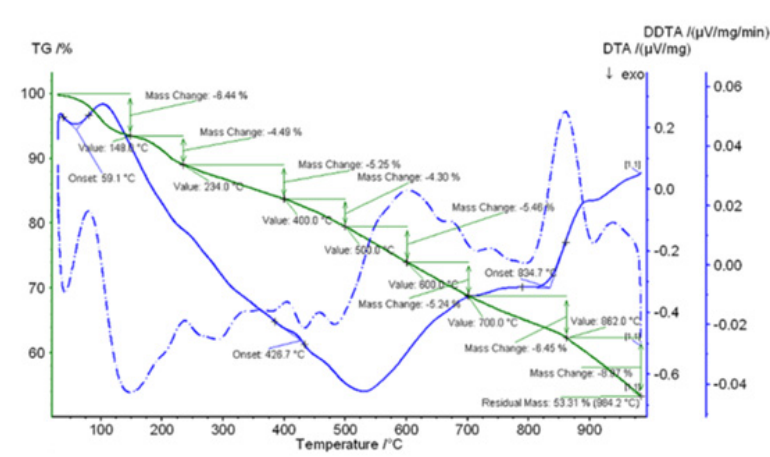

CM-95-NS

Figure 5. DTA analysis of different CNT obtained in this study

To obtain further information, TGA was performed. Figure 5 shows a comparison of the mass losses of CM-95 and oxidized CM-95. 


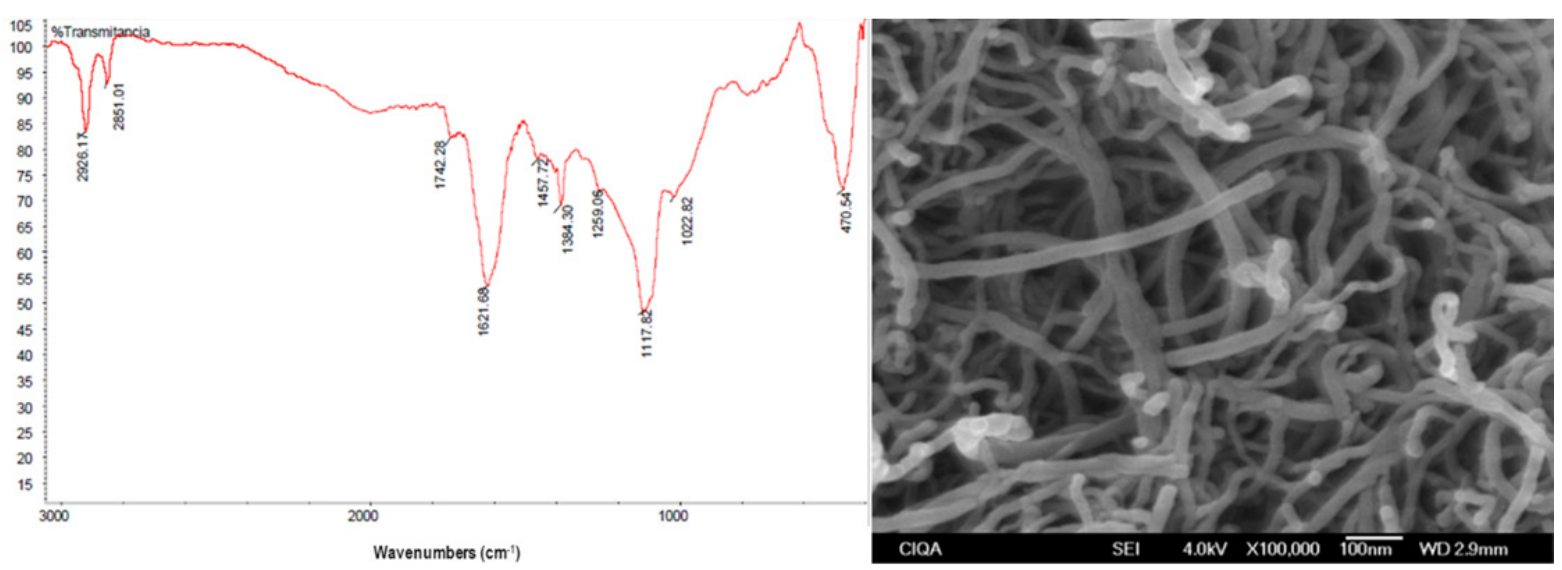

Figure 6. Infrared spectrum and SEM image of CM-95-NS

Figure 6 shows the infrared spectrum of CM-95-NS and its corresponding SEM image. SEM image shows a wall roughness on oxidized MWCNTs, meanwhile the characteristic bands of carbonyl groups at $1745 \mathrm{~cm}^{-1}$ can be seen in the infrared spectrum implying oxidation by treatment with nitric acid. Consequently, it is assumed that the surface roughness in CM-95-NS was produced by wall involvement due to oxidative conditions with $\mathrm{HNO}_{3}$. CM-95-G did not show a marked surface roughness; however, there are areas suggesting van der Waals interactions between the (3-glycidyloxypropyl)trimethoxysilane and CM-95. This is consistent with the values of average diameter of $21.1 \mathrm{~nm}$ and $3.5 \%$ loss in weight at TGA (Table 3). Similar observations can be inferred for CM-95-O and CM-95-NSO.

Additionally, there is little compatibility between trimethoxy (octyl) silane and CM-95, suggested by the average diameter of 16.5 and $26.2 \mathrm{~nm}$ respectively (Table 3). By comparison of results of average diameter and weight loss, it can be concluded that CM-95-NSO samples are only coated with triethoxy (octyl) silane via Van der Waals forces. This is supported by the $14.6 \%$ weight loss results between $250{ }^{\circ} \mathrm{C}$ and $450{ }^{\circ} \mathrm{C}$ obtained via TGA and the average diameter of $26.2 \mathrm{~nm}$; compared with the weight loss showed by CM-95-NSG of $7.9 \%$ and an average diameter of $13.4 \mathrm{~nm}$, which indicates a covalent chemical bond between CM-95-NS and (3-glycidyloxypropyl) trimethoxysilane.

Table 3. Average diameter by SEM and weight loss by TGA of functionalized CNTs

\begin{tabular}{lcc}
\hline \multicolumn{1}{c}{ Sample } & $\begin{array}{c}\text { Average Diameter in } \\
\text { SEM (nm) }\end{array}$ & $\begin{array}{c}\text { Weight loss in TGA, } \\
250-4500^{\circ} \mathrm{C}(\%)\end{array}$ \\
\hline CM-95 & 13.7 & 1.6 \\
CM-95-NS & 15.8 & 7.4 \\
CM-95-G & 21.1 & 3.5 \\
CM-95-NSG & 13.4 & 7.9 \\
CM-95-O & 16.5 & 4.8 \\
CM-95-NSO & 26.2 & 14.6 \\
\hline
\end{tabular}

\subsection{Composites}

By comparing results shown in Table 4 and Table 5, it can be infered a dependency between nanotubes diameter and the functionalizing reagent, along with its dispersion in PMMA. For example, CM-95-NSO has more affinity with PMMA and forms bonds with van der Waals forces, therefore showing the highest average diameter of $37.2 \mathrm{~nm}$.

Figure 7 shows the SEM images of CNTs dispersion in PMMA polymer matrix composites, which showed an apparently homogeneous dispersion; even though small-entrapped voids and bubbles were clearly observed. It also shows the alignment and dispersion of CM-95, CM-95-G and CM-95-NSO in PMMA, with CM-95-NSO/PMMA (2/98) showing the best dispersion. Table 5 presents the data obtained by TGA 
thermograms of PMMA with CNT. It can be seen that the samples showed two phases of main decomposition $30-600{ }^{\circ} \mathrm{C}$, although some of them have low percentage decomposition between 250 and $300{ }^{\circ} \mathrm{C}$. The carbonaceous residue, product of the decomposition of the material, shows variations that suggest effects of mechanical dispersion and magnetic field on the nanotubes.

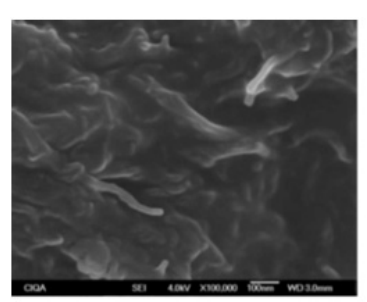

CM-95/PMMA (5/95)

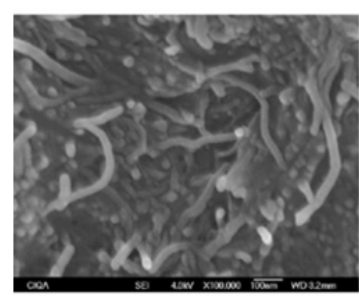

CM-95/PMMA (5/95) under magnetic field

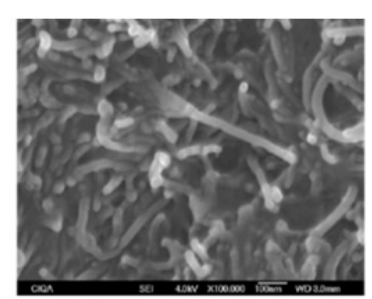

CM-95-G/PMMA (5/95)

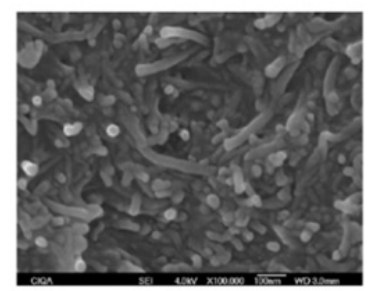

CM-95-G/PMMA (5/95) under magnetic field

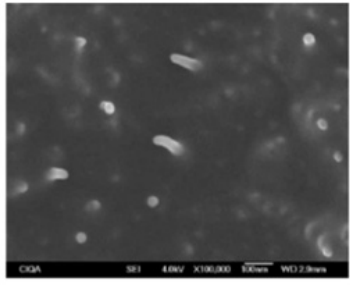

CM-95-NSO/PMMA (2/98)

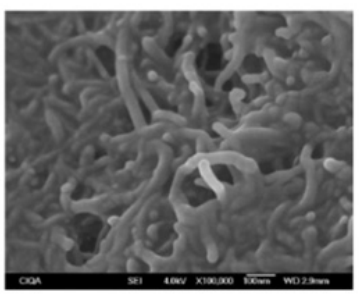

CM-95-NSO/PMMA (2/98) under magnetic field

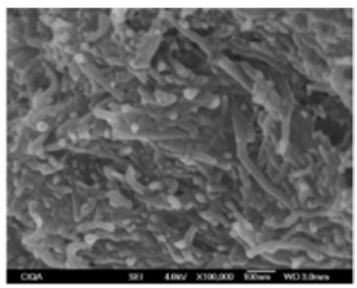

CM-95-NSG/PMMA (5/95)

Figure 7. SEM analysis of composites

Table 4. Carbon nanotubes average diameter in PMMA

\begin{tabular}{lcc}
\hline \multicolumn{1}{c}{ Sample } & Average diameter $(\mathrm{nm})$ & $\begin{array}{c}\text { Average diameter under magnetic } \\
\text { field }(\mathrm{nm})\end{array}$ \\
\hline CM-95/PMMA & 27.6 & 32.7 \\
CM-95-NSG/PMMA & 30.6 & ----- \\
CM-95-NSO/PMMA & 37.2 & 37.4 \\
CM-95-G/PMMA & 32.8 & 36.9 \\
\hline
\end{tabular}

Table 5. TGA composition analysis

\begin{tabular}{|c|c|c|c|c|c|}
\hline Sample & $\begin{array}{c}1^{\text {st }} \text { phase } \\
(\%)\end{array}$ & $\begin{array}{c}2^{\text {nd }} \text { phase } \\
(\%)\end{array}$ & $\begin{array}{c}3^{\text {th }} \text { phase } \\
(\%)\end{array}$ & $\begin{array}{c}\text { Carbonous } \\
\text { residue } \\
(\%)\end{array}$ & $\begin{array}{c}\text { Inorganic } \\
\text { material } \\
(\%)\end{array}$ \\
\hline CM-95/PMMA & $\begin{array}{c}159.63{ }^{\circ} \mathrm{C} \\
(8.87)\end{array}$ & ---- & $\begin{array}{c}378.3^{\circ} \mathrm{C} \\
(84.92)\end{array}$ & 5.05 & 1.15 \\
\hline $\begin{array}{l}\text { CM-95/PMMA } \\
\text { Magnetic field }\end{array}$ & $\begin{array}{c}143.78{ }^{\circ} \mathrm{C} \\
(11.25)\end{array}$ & ---- & $\begin{array}{c}375.17^{\circ} \mathrm{C} \\
(83.05)\end{array}$ & 4.40 & 1.27 \\
\hline CM-95-G/PMMA & $\begin{array}{c}163.86^{\circ} \mathrm{C} \\
(8.93)\end{array}$ & $\begin{array}{c}292.76^{\circ} \mathrm{C} \\
(2.23)\end{array}$ & $\begin{array}{c}382.57^{\circ} \mathrm{C} \\
(80.35)\end{array}$ & 7.40 & 1.07 \\
\hline CM-95-NSG/PMMA & $\begin{array}{c}144.8^{\circ} \mathrm{C} \\
(10.39)\end{array}$ & $\begin{array}{c}286.4^{\circ} \mathrm{C} \\
(2.80)\end{array}$ & $\begin{array}{c}377.28^{\circ} \mathrm{C} \\
(86.15)\end{array}$ & ---- & 0.65 \\
\hline CM-95-NSO/PMMA & $\begin{array}{c}125.82{ }^{\circ} \mathrm{C} \\
(11.34)\end{array}$ & ---- & $\begin{array}{c}374.11^{\circ} \mathrm{C} \\
(87.37)\end{array}$ & ---- & 1.27 \\
\hline $\begin{array}{l}\text { CM-95-NSO/PMMA } \\
\text { Magnetic field }\end{array}$ & $\begin{array}{c}115.26^{\circ} \mathrm{C} \\
11.51\end{array}$ & ---- & $\begin{array}{c}372.0^{\circ} \mathrm{C} \\
84.66\end{array}$ & 2.95 & 0.87 \\
\hline
\end{tabular}


The molecular mechanical and quantum mechanical calculations developed a MOPAC theory PM6. The highest occupied molecular orbital (HOMO) and lowest unoccupied molecular orbital (LUMO) are indicated in Table 6.

Table 6. Molecular orbital theory: total, high (HOMO) and low (LUMO) atomic orbital energies

\begin{tabular}{lccc}
\hline \multicolumn{1}{c}{ Fragment } & Total Energy $\times 10^{-3}(\mathrm{eV})$ & HOMO Energy $\times 10^{-1}(\mathrm{eV})$ & LUMO Energy $(\mathrm{eV})$ \\
\hline Ketone function & -1.978 & -0.907 & 0.28 \\
No Functionalized & -1.986 & -0.829 & 0.43 \\
Epoxy function & -2.276 & -0.903 & 0.10 \\
Carboxyl function & -2.298 & -0.887 & 0.43 \\
Ether function & -2.305 & -0.882 & 0.50 \\
Hydroxyl function & -2.306 & -0.876 & 0.55 \\
$-\mathrm{NO}_{2}$ function & -2.760 & -0.922 & -0.11 \\
\hline
\end{tabular}

Here, first-principles calculations were used to investigate the fundamental molecular interactions in a representative CM-95 and $\mathrm{HNO}_{3} / \mathrm{H}_{2} \mathrm{SO}_{4}$ system. Results show that $\mathrm{CM}-95$ oxidation state has profound effects on the most stable chemical states and arrangements of the system (Figure 8). Furthermore, the energetic trends of the various chemical states depicted in Table 6 are consistent with what has been suggested from the experimental observations. These results justify the idea that, in agreement with literature (Kanai, Khalap, Collins, \& Grossman, 2010), control of the oxidation state can effectively direct molecular functionalization of a CNT sidewall.
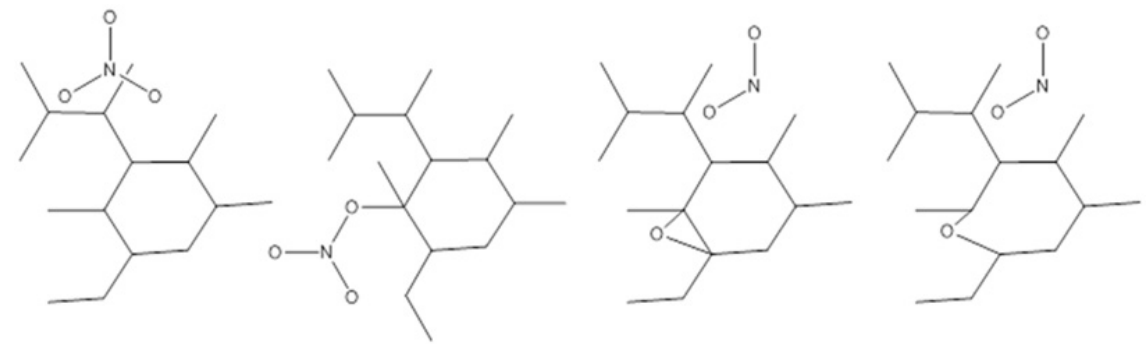

Figure 8. Four chemical states investigated here include, from left to right, physisorbed, chemisorbed, epoxide-incorporated and ether-configurations. Charge transfer between the molecule and CM-95 is shown for the physisorbed and chemisorbed states

\section{Conclusions}

To improve the load transfer in composites between the matrix and filler a covalent linking between the two components is desirable. One approach to solve these problems is functionalization of the CNT source material prior to its incorporation into the polymer matrix. Oxidation of MWCNTs was performed and supported by disorder and defects in the carbon lattice and change in the amount and type of chemical groups attached to the oxidized MWCNTs walls, shown in Raman spectroscopy and FTIR. Also, roughness areas are visible in SEM images indicating Van der Waals interactions between CNTs and functionalization reagents. Composites showed good dispersion of filler; with the best one for oxidized-silane functionalized CNTs. Carbonaceous residue suggests effects of mechanical dispersion and magnetic field on the nanotubes. The discrepancies between different approaches result from several sources, like the sensitivity and accuracy of the experimental and characterization methods applied and assumptions of models evaluating the chemical and physical quantities. For example, Raman and FTIR spectroscopy do not quantify the bulk content of Carbon $\mathrm{sp}^{2} / \mathrm{sp}^{3}$ and other chemical carbon-oxygen-hydrogen groups, but indicate and compare qualitatively the existing groups after different sample treatment. The dissociative incorporation of an oxygen atom into the MWCNTs sidewall becomes highly favorable when the CNTs oxidation state is elevated from electron density depletion in the vicinity, as caused experimentally using electrochemical potential. The elevation of the CNTs oxidation state 
through accumulating local charge transfer from the surrounding molecules does not have the same effect. Amount of surface groups depends on the morphology and structure of CNT increasing with increase of nanotubes' defectiveness. Compatibility and solubility continue to be an issue, and new purification and characterization techniques are still needed.

\section{References}

Ajayan, P. M. (1999). Nanotubes from carbon. Chemical Reviews, 99(7), 1787-1800. http://dx.doi.org/10.1021/cr970102g

Amorim, R. G., Fazzio, A., Antonelli, A., Novaes, F. D., \& da Silva, A. J. R. (2007). Divacancies in graphene and carbon nanotubes. Nano Letters, 7(8), 2459-2462. http://dx.doi.org/10.1021/n1071217v

Bachtold, A., Hadley, P., Nakanishi, T., \& Dekker, C. (2001). Logic circuits with carbon nanotube transistors. Science, 294(5545), 1317-1320. http://dx.doi.org/10.1126/science.1065824

Barraza, H. J., Pompeo, F., O'Rea, E. A., \& Resasco, D. E. (2002). SWNT-filled thermoplastic and elastomeric composites prepared by miniemulsion polymerization. Nano Letters, 2(8), 797-802. http://dx.doi.org/10.1021/n10256208

Chan, S. P., Chen, G., Gong, X. G., \& Liu, Z. F. (2003). Oxidation of carbon nanotubes by singlet $\mathrm{O}_{2}$. Physical Review Letters, 90(8), 086403. http://dx.doi.org/10.1103/PhysRevLett.90.086403

Chen, J., Hamon, M. A., Hu, H., Chen, Y., Rao, A. M., Eklund, P. C., \& Haddon, R. C. (1998). Solution properties of single-walled carbon nanotubes. Science, 282(5386), 95-98. http://dx.doi.org/10.1126/science.282.5386.95

Chiu, P. W., Duesberg, G. S., Dettlaff-Weglikowska, U., \& Roth, S. (2002). Interconnection of carbon nanotubes by chemical functionalization. Applied Physics Letters, 80(20), 3811-3813. http://dx.doi.org/10.1063/1.1480487

Collins, P. G., Bradley, K., Ishigami, M., \& Zettl, A. (2000). Extreme oxygen sensitivity of electronic properties of carbon nanotubes. Science, 287(5459), 1801-1804. http://dx.doi.org/10.1126/science.287.5459.1801

Curran, S. A., Talla, J. A., Zhang, D., \& Carroll, D. L. (2005). Defect-induced vibrational response of multi-walled carbon nanotubes using resonance Raman spectroscopy. Journal of Materials Research, 20(12), 3368-3373. http://dx.doi.org/10.1557/jmr.2005.0414

Du, F., Fischer, J. E., \& Winey, K. I. (2003). Coagulation method for preparing single-walled carbon nanotube/poly (methyl methacrylate) composites and their modulus, electrical conductivity, and thermal stability. Journal of Polymer Science Part B: Polymer Physics, 41(24), 3333-3338. http://dx.doi.org/10.1002/polb.10701

Giannozzi, P., Car, R., \& Scoles, G. (2003). Oxygen adsorption on graphite and nanotubes. The Journal of Chemical Physics, 118, 1003. http://dx.doi.org/10.1063/1.1536636

Goldberg-Oppenheimer, P., Eder, D., \& Steiner, U. (2011). Carbon nanotube alignment via electrohydrodynamic patterning of nanocomposites. Advanced Functional Materials, 21(10), 1895-1901. http://dx.doi.org/10.1002/adfm.201002692

Gomez-Navarro, C., De Pablo, P. J., Gómez-Herrero, J., Biel, B., Garcia-Vidal, F. J., Rubio, A., \& Flores, F. (2005). Tuning the conductance of single-walled carbon nanotubes by ion irradiation in the Anderson localization regime. Nature Materials, 4(7), 534-539. http://dx.doi.org/10.1038/nmat1414

Hill, D. E., Lin, Y., Rao, A. M., Allard, L. F., \& Sun, Y.-P. (2002). Functionalization of carbon nanotubes with polystyrene. Macromolecules, 35(25), 9466-9471. http://dx.doi.org/10.1021/ma020855r

Holzinger, M., Vostrowsky, O., Hirsch, A., Hennrich, F., Kappes, M., Weiss, R., \& Jellen, F. (2001). Sidewall functionalization of carbon nanotubes. Angewandte Chemie International Edition, 40(21), 4002-4005. http://dx.doi.org/10.1002/1521-3773(20011105)40:21<4002::AID-ANIE4002>3.0.CO;2-8

Jhi, S. H., Louie, S. G., \& Cohen, M. L. (2005). Reentrant semiconducting behavior of zigzag carbon nanotubes at substitutional doping by oxygen dimers. Physical Review Letters, 95(22), 226403. http://dx.doi.org/10.1103/PhysRevLett.95.226403

Kanai, Y., Khalap, V. R., Collins, P. G., \& Grossman, J. C. (2010). Atomistic oxidation mechanism of a carbon nanotube in nitric acid. Physical Review Letters, 104(6), 066401. http://dx.doi.org/10.1103/PhysRevLett.104.066401 
Kimura, T., Ago, H., Tobita, M., Ohshima, S., Kyotani, M., \& Yumura, M. (2002). Polymer composites of carbon nanotubes aligned by a magnetic field. Advanced Materials, 14(19), 1380-1383. http://dx.doi.org/10.1002/1521-4095(20021002)14:19<1380::AID-ADMA1380>3.0.CO;2-V

Klopman, G. (1974). Chemical reactivity and reaction paths. Wiley.

Kondratyuk, P., \& Yates Jr, J. T. (2004). Nanotubes as molecular sponges: the adsorption of $\mathrm{CCl}_{4}$. Chemical Physics Letters, 383(3), 314-316. http://dx.doi.org/10.1016/j.cplett.2003.10.151

Leonard, F. (2008). Physics of carbon nanotube devices. Elsevier Science.

Li, Y.-H., Wang, S., Luan, Z., Ding, J., Xu, C., \& Wu, D. (2003). Adsorption of cadmium (II) from aqueous solution by surface oxidized carbon nanotubes. Carbon, 41(5), 1057-1062. http://dx.doi.org/10.1016/S0008-6223(02)00440-2

Ma, C. G., Wang, Y. R., \& Yu, Y. J. (2010). Electrical and thermal properties of carbon nanotubes/PMMA composites induced by low magnetic fields. Plastics, Rubber and Composites, 39(2), 49-53. http://dx.doi.org/10.1179/174328910X12608851832371

Mazov, I., Kuznetsov, V. L., Simonova, I. A., Stadnichenko, A. I., Ishchenko, A. V., Romanenko, A. I., ... Anikeeva, O. B. (2012). Oxidation behavior of multiwall carbon nanotubes with different diameters and morphology. Applied Surface Science, 258(17), 6272-6280. http://dx.doi.org/10.1016/j.apsusc.2012.03.021

Meyer, M., Etchegoin, P. G., \& Le Ru, E. C. (2010). Electronic structure, Raman tensors, and resonance phenomena in a simple molecular model. American Journal of Physics, 78, 300. http://dx.doi.org/10.1119/1.3271796

Murugesh, A. K., Uthayanan, A., \& Lekakou, C. (2010). Electrophoresis and orientation of multiple wall carbon

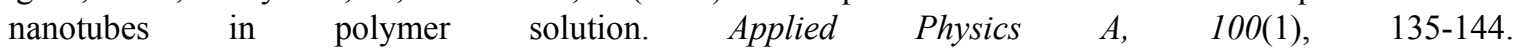
http://dx.doi.org/10.1007/s00339-010-5562-y

Pumera, M. (2009). The electrochemistry of carbon nanotubes: fundamentals and applications. Chemistry-A European Journal, 15(20), 4970-4978. http://dx.doi.org/10.1002/chem.200900421

Rueckes, T., Kim, K., Joselevich, E., Tseng, G. Y., Cheung, C. L., \& Lieber, C. M. (2000). Carbon nanotube-based nonvolatile random access memory for molecular computing. Science, 289(5476), 94-97. http://dx.doi.org/10.1126/science.289.5476.94

Shim, M., Back, J. H., Ozel, T., \& Kwon, K.-W. (2005). Effects of oxygen on the electron transport properties of carbon nanotubes: Ultraviolet desorption and thermally induced processes. Physical Review B, 71(20), 205411. http://dx.doi.org/10.1103/PhysRevB.71.205411

Stobinski, L., Lesiak, B., Kövér, L., Tóth, J., Biniak, S., Trykowski, G., \& Judek, J. (2010). Multiwall carbon nanotubes purification and oxidation by nitric acid studied by the FTIR and electron spectroscopy methods. Journal of Alloys and Compounds, 501(1), 77-84. http://dx.doi.org/10.1016/j.jallcom.2010.04.032

Sumanasekera, G. U., Pradhan, B. K., Romero, H. E., Adu, K. W., \& Eklund, P. C. (2002). Giant thermopower effects from molecular physisorption on carbon nanotubes. Physical Review Letters, 89(16), 166801. http://dx.doi.org/10.1103/PhysRevLett.89.166801

Sun, Y.-P., Fu, K., Lin, Y., \& Huang, W. (2002). Functionalized carbon nanotubes: properties and applications. Accounts of Chemical Research, 35(12), 1096-1104. http://dx.doi.org/10.1021/ar010160v

Tans, S. J., Verschueren, A. R. M., \& Dekker, C. (1998). Room-temperature transistor based on a single carbon nanotube. Nature, 393(6680), 49-52. http://dx.doi.org/10.1038/29954

Terrones, M., Terrones, H., Banhart, F., Charlier, J. C., \& Ajayan, P. M. (2000). Coalescence of single-walled carbon nanotubes. Science, 288(5469), 1226-1229. http://dx.doi.org/10.1126/science.288.5469.1226

Wang, Z., Shirley, M. D., Meikle, S. T., Whitby, R. L. D., \& Mikhalovsky, S. V. (2009). The surface acidity of acid oxidised multi-walled carbon nanotubes and the influence of in-situ generated fulvic acids on their stability in aqueous dispersions. Carbon, 47(1), 73-79. http://dx.doi.org/10.1016/j.carbon.2008.09.038

Wildgoose, G. G., Banks, C. E., Leventis, H. C., \& Compton, R. G. (2006). Chemically modified carbon nanotubes for use in electroanalysis. Microchimica Acta, 152(3-4), 187-214. http://dx.doi.org/10.1007/s00604-005-0449-x

Yao, Z., Postma, H. W. C., Balents, L., \& Dekker, C. (1999). Carbon nanotube intramolecular junctions. Nature, 402(6759), 273-276. http://dx.doi.org/10.1038/46241 
Zeng, Y., Ci, L., Carey, B. J., Vajtai, R., \& Ajayan, P. M. (2010). Design and reinforcement: vertically aligned carbon nanotube-based sandwich composites. ACS Nano, 4(11), 6798-6804. http://dx.doi.org/10.1021/nn101650p

Zhao, J., Lu, J. P., Han, J., \& Yang, C. K. (2003). Noncovalent functionalization of carbon nanotubes by aromatic organic molecules. Applied Physics Letters, 82(21), 3746-3748. http://dx.doi.org/10.1063/1.1577381

Zhou, T., Wu, J., Duan, W., \& Gu, B.-L. (2007). Physical mechanism of transport blocking in metallic zigzag carbon nanotubes. Physical Review B, 75(20), 205410. http://dx.doi.org/10.1103/PhysRevB.75.205410

\section{Copyrights}

Copyright for this article is retained by the author(s), with first publication rights granted to the journal.

This is an open-access article distributed under the terms and conditions of the Creative Commons Attribution license (http://creativecommons.org/licenses/by/3.0/). 